\title{
Investigation of Early Degradation Processes at Coated Metals by AC-Scanning Electrochemical Microscopy
}

\author{
J. J. Santana ${ }^{\mathrm{a}, \mathrm{b}}$, R. M. Souto ${ }^{\mathrm{a}}$, S. González ${ }^{\mathrm{a}}$ M. Pähler ${ }^{\mathrm{c}}$ and W. Schuhmann ${ }^{\mathrm{c}}$ \\ a Department of Physical Chemistry, University of La Laguna, E-38200 La Laguna, \\ Tenerife, Canary Islands, Spain \\ ${ }^{\mathrm{b}}$ Department of Process Engineering, University of Las Palmas de Gran Canaria, E-35017 \\ Las Palmas de Gran Canaria, Canary Islands, Spain \\ ${ }^{\mathrm{b}}$ Analytische Chemie - Elektroanalytik \& Sensorik; Ruhr-Universität Bochum, \\ Universitätsstraße 150, D-44780 Bochum, Germany
}

\begin{abstract}
.
Alternating-current scanning electrochemical microscopy (AC-SECM) is employed to characterize the early stages of the degradation reactions occurring in metal-coating systems upon exposure to an aqueous environment. The spatial resolution of the technique results from the measurement of changes in the resistance of the thin electrolyte layer comprised between the tip and the surface of the coating due to variations in the tipsubstrate distance. Resistance measurements are conducted at various frequencies of the AC perturbation signal effectively allowing topographic changes to be monitored as a function of time. Furthermore, AC-SECM can be used to determine the tip-substrate distance without the addition of redox mediators to the electrolyte, which might affect the chemical properties of the system. In this way, the effect of chloride ions from the aqueous phase to induce either the heterogeneous absorption of water by the coating, or its accumulation at the metal-substrate interface, has been imaged.
\end{abstract}

\section{Introduction}

The application of organic films on metallic materials is the most widely employed technique for their corrosion protection. Anticorrosion paints and inhibitors produce a physical barrier against the diffusion of corrosive agents from the electrolytic phase to the surface of the metal [1-4]. Yet, a perfect barrier layer is never attained because diffusion of water and oxygen always occurs upon exposure of a coated metal to the aqueous phase [1].

The electrochemical nature of the aqueous corrosion reactions has led to the application of electrochemical techniques to monitor these processes, and among them, electrochemical impedance spectroscopy (EIS) is widely employed [1,5-9]. Unfortunately, electrochemical techniques do not have spatial resolution as they average the behaviour of the exposed sample, thus making difficult to gain relevant information on the early stages of degradation processes such as blistering which occur at localized sites in the metal-coating interface. A new situation has arisen from the development of the Scanning Electrochemical Microscope (SECM) by Bard and coworkers two decades ago [10]. This method is based upon the measurement of a faradaic current at an ultramicroelectrode tip that is scanned over the surface of a material. In the feedback mode, the measured current results from the oxidation/reduction of an electrochemically-active substance in the liquid phase (i.e., redox mediator) [10-12], which is greatly affected by the tip-substrate distance. In the case of a non-defective coating, the current measured at the tip will be affected by the extent of the blocking effect produced by the surface of the coating to the diffusion of the redox mediator towards the tip [13]. In this way, changes in the topography of the coating upon immersion in an aqueous environment can be monitored as a function of time. The 
formation of bulges under the coating in chloride-containing solutions has thus been found [13-17], which might be regarded as the early stages of blistering. This effect has also been observed to be affected by the composition of the electrolyte, particularly in relation to chloride ions, whereas no heterogeneous water uptake occurred in sulphate, nitrate and borate-based environments [13,15].

Despite the potential of SECM to investigate coated metals with high spatial resolution, the technique relies on the use of a redox mediator which must be added to the electrolyte phase. Unfortunately, this redox system can modify the electric state of the working system (e.g. the potential at the interface will be controlled by the ratio of oxidized/reduced species as given by the corresponding Nernst equation), and/or may eventually diffuse in the polymeric matrix, leading to changes in either the concentration of the redox species in the electrolyte or the modification of the chemistry in the coating. In the case of these events, the tip-substrate distance cannot be established, and the system may not be operating at its spontaneous open circuit potential. These drawbacks can be avoided by using the SECM in the alternating current mode (AC-SECM) [18-24], because the operating principle of the technique does not involve the use of a redox mediator for imaging.

For an SECM tip located in the proximity of an insulating surface such as an organic coating, the AC electric current flowing between the auxiliary and the tip electrodes must be conducted through the electrolyte solution (see Figure 1-a). The equivalent circuit for this system contains a faradaic impedance at the tip occurring in parallel to a condenser that accounts for the electric double-layer developed at the tip-electrolyte interface. These two components are in series with the ohmic resistance of the electrolyte as shown in Figure 1-b.

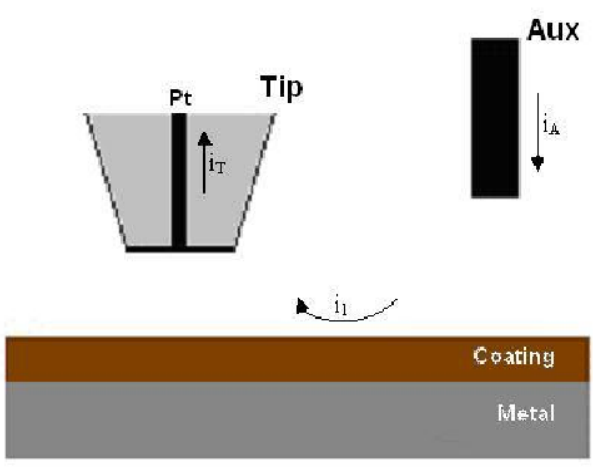

(a)

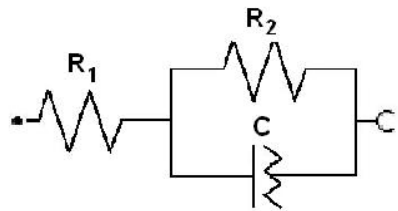

(b)

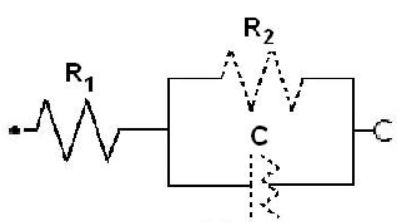

(c)

Figure 1: (a) Sketch depicting the electrochemical system formed by the tip, an insulating substrate and the auxilliary electrode. (b) Equivalent circuit for the system, where $R_{1}$ is the electrolyte resistance, $R_{2}$ is the tip resistance, and $C$ the capacitance at the tip. (c) At high AC frequencies, the capacitance leaks and the impedance of the system is given by the element $R_{1}$.

As the tip approaches the insulating surface, the resistance of the electrolyte comprised between the tip and the substrate increases because the layer becomes thinner. Simultaneously, there is an increase in the capacitance of the double layer developed at the tip-electrolyte interface. By considering the resistance of the tip to be either constant or negligible compared to the other impedance components in this equivalent circuit, a relationship can be established between the electrolyte resistance and the tip-substrate distance, and it matches the typical relationship between the tip current and the tip-substrate distance governing conventional SECM, namely: 


$$
\frac{R(\infty)}{R(d)}=\frac{i_{T}(d)}{i_{T}(\infty)}
$$

where $i_{T}(d)$ and $R(d)$ are the feedback current and the solution resistance, respectively, at a normalized distance $d$ of the tip from the substrate. From the introduction of the angular frequency and the amplitude of the AC potential signal in the equations describing the equivalent circuit under consideration, an equation for the normalized solution resistance can be derived:

$$
\omega C_{d l} R(d)=\frac{i_{0}=(d)}{i_{90}=(d)}
$$

where $\omega$ is the angular frequency, $C_{d l}$ is the double layer capacitance, $R$ is the solution resistance, and $i$ is the current measured at the tip. Depending on the electrochemical characteristics of the substrate, the current will flow through the pathway of smaller impedance at each frequency. Furthermore, at a given frequency, the analysis of either the magnitude or the phase shift of the AC current can be related to the topography of the insulating substrate provided the tip is scanned at a constant height. To this end, a poorlyconductive electrolyte (namely, $1 \mathrm{mM} \mathrm{Na}_{2} \mathrm{SO}_{4}$ ) has been employed to image by AC-SECM the evolution of the surface topography of a coil-coated steel after exposed to $3 \mathrm{wt} \% \mathrm{NaCl}$.

\section{Experimental}

This work was carried out on a coil-coated steel (CCS) system consisting in a $0.4 \mathrm{~mm}$ mild steel foil coated with a $20 \mu \mathrm{m}$ galfan ( $\mathrm{Zn}-5 \mathrm{Al})$ coating, and this in turn being covered with $25 \mu \mathrm{m}$ white polyester paint (including $5 \mu \mathrm{m}$ primer and $20 \mu \mathrm{m}$ top coat). The CCS panels were cut to the desired size with a guillotine from the top coat side downwards, and measured $30 \mathrm{~mm}$ x $25 \mathrm{~mm}$. These coupons were placed horizontally in a flat-cell configuration at the bottom of the AC-SECM cell.

The set-up and imaging procedures for AC-SECM have been described elsewhere [2527]. The instrument consisted of a step-motor-driven $\mathrm{x}, \mathrm{y}, \mathrm{z}$ stage (Owis $\mathrm{GmbH}$, Staufen, Germany) with a resolution of $0.625 \mathrm{~mm}$ per half step and $48 \mathrm{~nm}$ per microstep. The electrochemical control was achieved using a PAR 273 potentiostat (Ametek, Oak Ridge, USA) with a bandwidth of $100 \mathrm{kHz}$ was used, and a Perkin-Elmer 7280 digital lock-in amplifier (Ametek, Oak Ridge, USA). The tips for the AC-SECM studies were glass insulated, disk-shaped Pt microelectrodes (25 $\mu \mathrm{m}$ disk diameter) [28].

The experiments were conducted in the three-electrode configuration, with the SECM tip as working electrode, a Pt-wire as counter, and a chlorinated silver wire as pseudoreference electrode, whereas the substrate was left unbiased, thus allowing the measurements to be carried out on the metal-coating system without any electrochemical perturbation. The sinusoidal voltage applied to the system was $100 \mathrm{mV}$ (peak to peak) in the frequency range from 351 to $55490 \mathrm{~Hz}$ with a total number of points of 26 (logarithmic spaced) with a potential amplitude of $0.1 \mathrm{~V}$ and a sensibility of $200 \mathrm{mV}$. The system AC response was analysed in terms of the phase shift $\theta$ of the AC current signal monitored. Approach curves ( $R$ vs. $d$ ) were recorded and used for tip positioning, whereas monitoring $\theta$ as a function of $x$ - and $y$-tip position allowed imaging. The operating tip-to-sample distance for imaging was set at $12 \mu \mathrm{m}$, and images of area $700 \mu \mathrm{m}$ x $700 \mu \mathrm{m}$ were obtained at a scan rate of $25 \mu \mathrm{m} \mathrm{s}^{-1}$. 
Testing of coated samples to examine the effects on blistering susceptibility was carried out in 3 wt.\% $\mathrm{NaCl}$ solution for selected immersion times. Since AC-SECM operation requires a low-conductivity electrolyte solution, measurements were performed in $1 \mathrm{mM}$ $\mathrm{Na}_{2} \mathrm{SO}_{4}$, which was regarded to be inert towards blister initiation under the coating [13]. Electrolyte-exchange was carefully performed in the small electrochemical cell between subsequent treatment and testing steps. The electrolytes were prepared using twice-distilled water and analytical grade reagents (Sigma-Aldrich, Deisenhofen, Germany).

\section{Results and discussion}

Figure 2 shows a selection of the approach curves measured at different frequencies of the AC signal as the tip is moved towards the surface of the coated metal in $1 \mathrm{mM} \mathrm{Na} 2 \mathrm{SO}_{4}$. This procedure allows for the tip-substrate distance to be established, as well as to determine the frequency at which the greatest resolution is achieved. In this work, the biggest values for the magnitude of the AC current, and the greatest variation with the tipsubstrate distance, were both observed at $4414 \mathrm{~Hz}$.

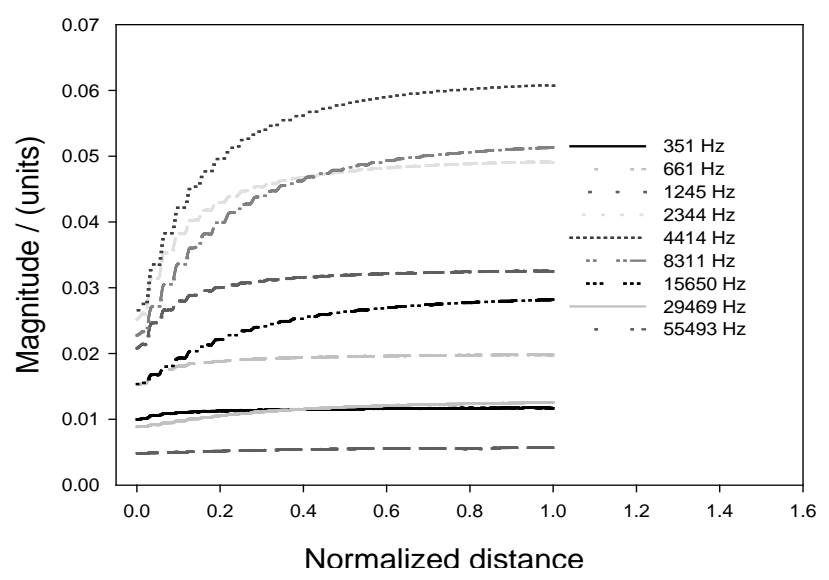

Figure 2: Normalized approach curves towards a painted sample immersed in $1 \mathrm{mM} \mathrm{Na} \mathrm{SO}_{4}$ measured with a $25 \mu \mathrm{m}$ diameter Pt electrode. The excitation signal amplitude was $100 \mathrm{mV}_{\mathrm{pp}}$ and the frequency range extended from 351 to $55493 \mathrm{~Hz}$. The sample was approached in the test electrolyte prior to its exposure in the $3 \mathrm{wt} \% \mathrm{NaCl}$ aqueous solution.

Once the approach curves were thus recorded, the tip was removed from the surface to a distance of $12 \mu \mathrm{m}, X-Y$ maps over the surface were recorded at a constant height using AC potential signals in the $351 \leq f \leq 55493 \mathrm{~Hz}$ frequency range. Replacement of the electrolyte was then performed to allow the coated surface to be exposed to the $3 \mathrm{wt}$ \% $\mathrm{NaCl}$ solution for selected immersion times to investigate a possible specific effect of chloride ions towards heterogeneous water uptake by the coating. AC-SECM monitoring was accomplished after electrolyte exchange to the measuring environment (1 $\left.\mathrm{mM} \mathrm{Na}_{2} \mathrm{SO}_{4}\right)$, and the same measurement sequence was completed to record both the approach curves and the XY maps. The procedure can be repeated several times by exchanging the electrolyte in the electrochemical cell in order to follow the time evolution of the surface modification induced by chloride ions.

A first indication of the time evolution of the system can be derived from the inspection of Figure 3, which gives the variations in the phase angle of the AC current response when the tip is located in a fixed position over the coated sample, measured before and after the system has been exposed to the chloride-containing solution for different times. That is, for a given position over the coated sample, the values of the phase component have been extracted from the maps obtained at each frequency (cf. Figures 4-6) for various exposures. 


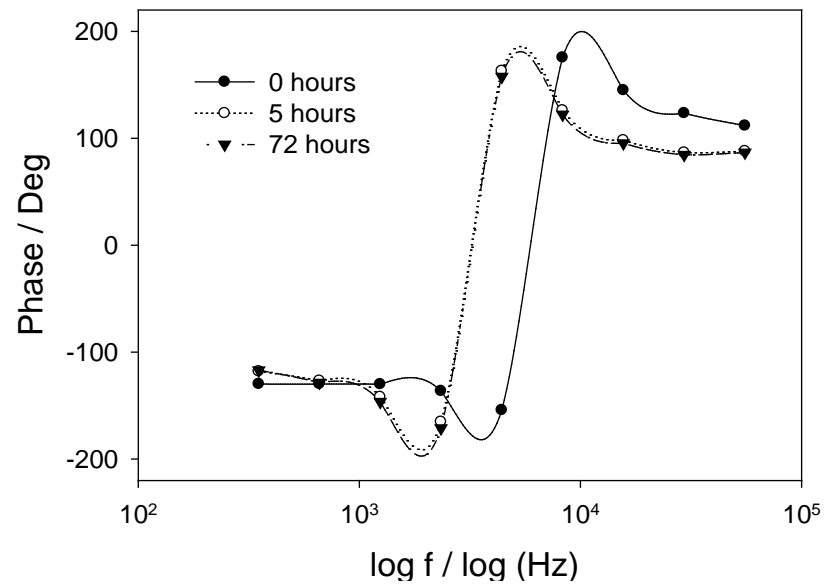

Figure 3: Frequency dependence of the phase component of the current response determined by AC-SECM when the tip is located over a cosen position over the coated sample. The phase values are determined from the images measured at different frequencies of the AC potential perturbation, and for different exposure periods of the simples to $3 \mathrm{wt} . \% \mathrm{NaCl}$ solution.

The initial condition of the coated sample corresponds to the measurement of negative values of the phase component for frequencies up to $44213 \mathrm{~Hz}$, whereas the change of sign occurs at a lower frequency for longer exposures. Indeed, the corresponding curves after immersion in $\mathrm{NaCl}$ solution merge together with independence of the duration of the exposures. The impedance for the equivalent circuit depicted in Figure 1-b is given by:

$$
Z=R_{1}+\frac{R_{2}}{1+\left(\omega R_{2} C\right)^{2}}+\frac{j\left(-\omega R_{2}^{2} C\right)}{1+\left(\omega R_{2} C\right)^{2}}
$$

where $Z$ denotes the total impedance expressed in ohms, $\omega(=2 \pi f)$ is the angular frequency, $j$ is the imaginary unit number, and $R_{1}, R_{2}$ and $C$ are the resistance and the capacitance components contained in the circuit. The real component of the impedance is represented by the first two terms in equation [3], whereas the remaining term corresponds to the imaginary part of the impedance. Accordingly, the phase angle is given by the reciprocal of the tangent given by the quotient between the imaginary and the real components of the impedance vector at each frequency. In the high frequency range, the only component in the equivalent circuit that will impose an opposition to time-varying electric current flowing through the cell is the ohmic resistance (see Figure 1-c). On the other hand, the ohmic resistance changes with the length of electrolyte volume comprised between the tip and the substrate, i.e., it varies with the tip-substrate distance. Since the AC-SECM images are measured at constant height of the tip over the coated substrate (namely $12 \mu \mathrm{m}$ ), topographic features developed on the surface of the coating will result in variations of the solution resistance, $R_{1}$. In this way, the value of the phase angle will be affected by this feature in the high frequency range. 

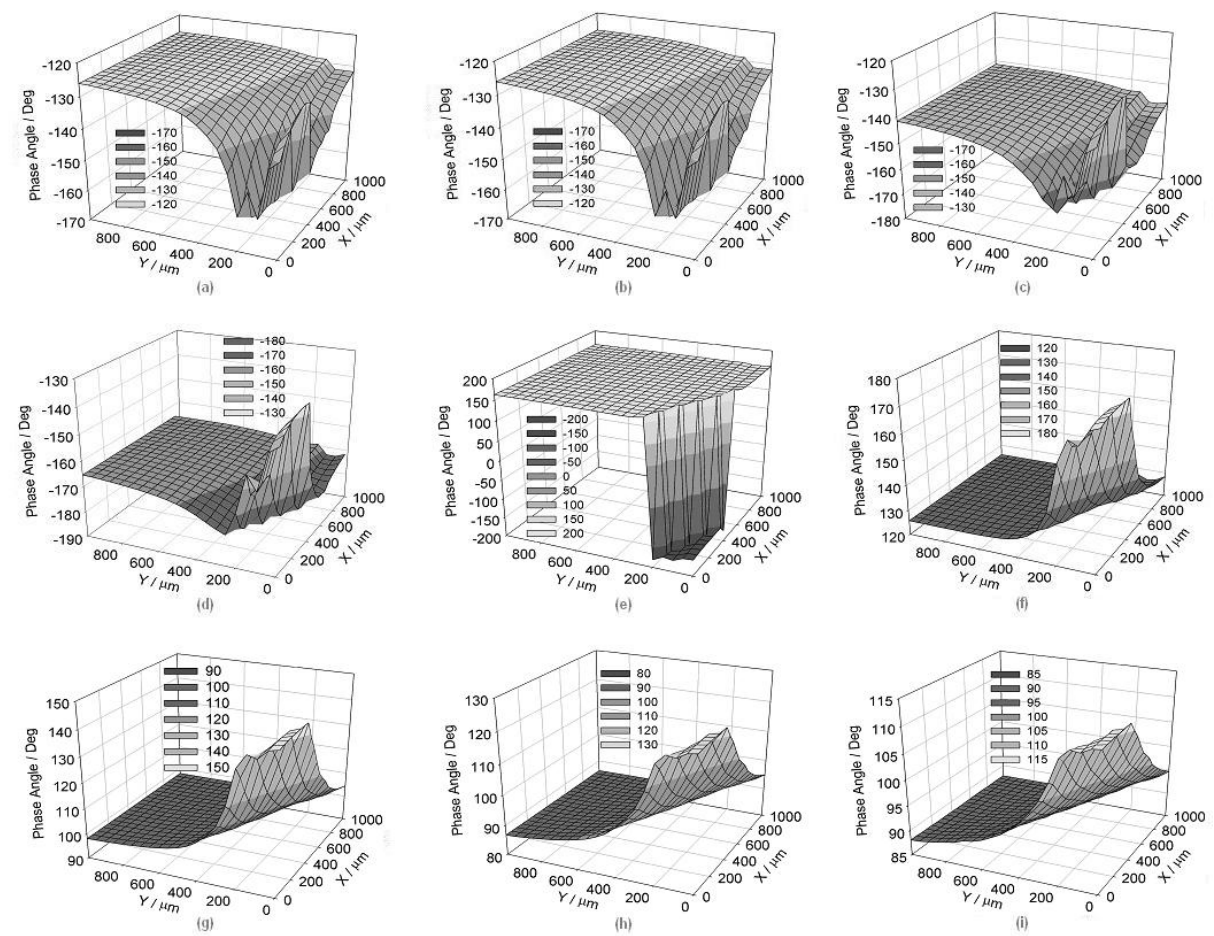

Figure 4: AC-SECM images of the coated sample upon immersion in $1 \mathrm{mM} \mathrm{Na}_{2} \mathrm{SO}_{4}$, prior to exposure to the chloride-containing solution (i.e., taken as $t=0$ ). The frequencies of the AC potential signal are: a) 351 , b) 660 , c) 1244 , d) 2343 , e) 4413 , f) 8310 , g) 15649 , h) 29469, and i) $55493 \mathrm{~Hz}$. Tip diameter: $10 \mu \mathrm{m}$; tip-substrate distance: $12 \mu \mathrm{m}$; $X$ and $Y$ step sizes: $50 \mu \mathrm{m}$.

A rather flat topography of the coating is shown by the AC-SECM images obtained for the coated sample before exposure to the chloride-containing solution (cf. Figure 4) regardless the frequency of the $\mathrm{AC}$ signal. For the selection of frequencies given in the figure, the change in the sign of the phase angle occurs between the maps corresponding 2342 and $4413 \mathrm{~Hz}$. Regardless the change of sign, the surface appears featureless in both cases for most of the scanned surface. Finally, the features appearing at the bottom left of the maps can be regarded as artefacts resulting from border effects. After the AC-SECM images were recorded in the measuring electrolyte, $1 \mathrm{mM} \mathrm{Na}_{2} \mathrm{SO}_{4}$, careful electrolyte exchange to $3 \mathrm{wt}$ \% $\mathrm{NaCl}$ solution (i.e. test electrolyte) was performed without removal of the coated sample in the cell. No AC-SECM measurements were conducted in the system during its immersion in the chloride-containing solution. After chosen exposure durations, electrolyte exchange was performed to introduce the measuring electrolyte $1 \mathrm{mM} \mathrm{Na} \mathrm{SO}_{4}$ back in the cell, and the same measurement procedures were employed as described above. 

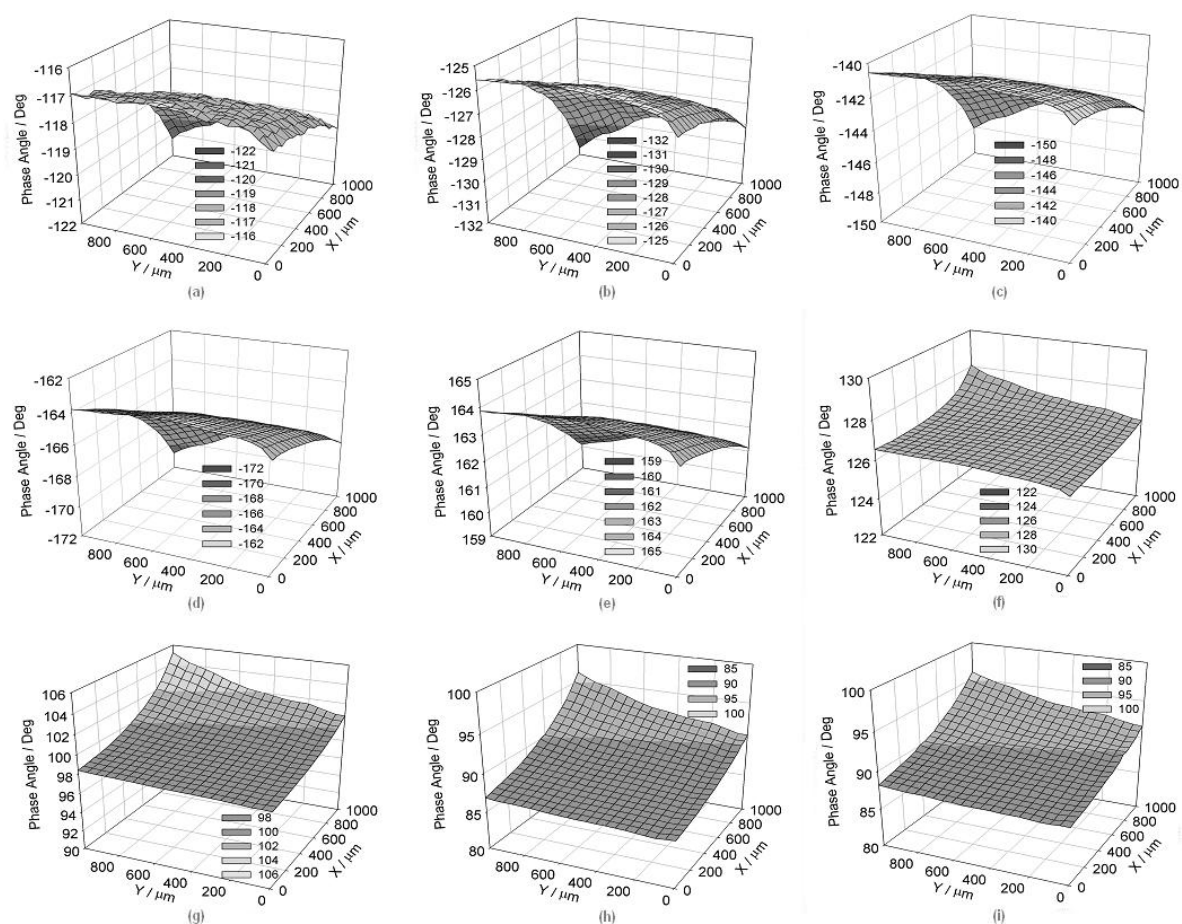

Figure 5: AC-SECM images of the coated sample upon immersion in $1 \mathrm{mM} \mathrm{Na}_{2} \mathrm{SO}_{4}$, after exposure to the chloride-containing solution for 5 hours. The frequencies of the AC potential signal are: a) 351, b) 660, c) 1244, d) 2343, e) 4413, f) 8310, g) 15649, h) 29469, and i) $55493 \mathrm{~Hz}$. Tip diameter: $10 \mu \mathrm{m}$; tip-substrate distance: $12 \mu \mathrm{m} ; X$ and $Y$ step sizes: $50 \mu \mathrm{m}$.

Figure 5 exhibits the AC-SECM images of the coating after exposure to $3 \mathrm{wt} . \% \mathrm{NaCl}$ solution for 5 hours. In this case, changes in the phase angle values measured over the simple are observed which are related to a variation of the distance between the tip and the substrate, though the tip height is maintained constant at $12 \mu \mathrm{m}$ during the whole scan. Thus, the development of topographic features in the surface are detected, which might be an early observation of blister or bulge formation in the coating due to electrolyte uptake after exposure in the chloride-containing solution. This effect becomes even more noticeable when the AC-SECM images obtained alter the simple was exposed to $3 \mathrm{wt} . \%$ $\mathrm{NaCl}$ solution for 72 hours as shown in Figure 6. In this case, the topographic features are more visible in the corresponding maps, thus confirming our assumption that chloride ions induce a specific effect during electrolyte uptake by the coating applied on the metallic substrate leading to the formation of a heterogeneous distribution of bulges, which may be regarded as the early stages of blister formation. And this effect occurs at very short exposures to the electrolyte, almost simultaneously to the initial water uptake by the coating. On the other hand, it has been shown that the high spatial resolution of the AC-SECM can be employed to visualize topographic changes occurring at coated samples related to degradation reactions. 

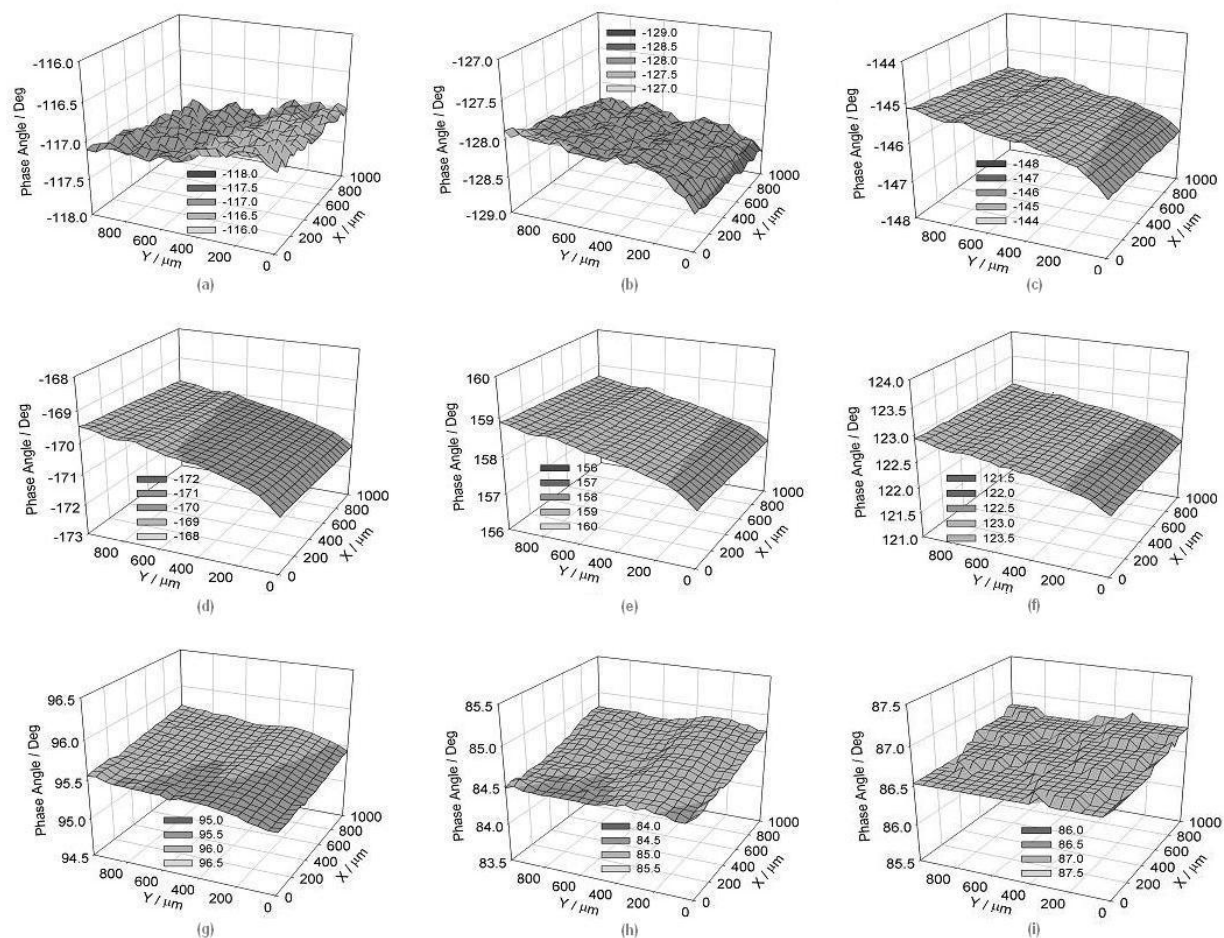

Figure 6: AC-SECM images of the coated sample upon immersion in $1 \mathrm{mM} \mathrm{Na}_{2} \mathrm{SO}_{4}$, after exposure to the chloride-containing solution for 72 hours. The frequencies of the AC potential signal are: a) 351, b) 660, c) 1244, d) 2343, e) 4413, f) 8310 , g) 15649 , h) 29469 , and i) $55493 \mathrm{~Hz}$. Tip diameter: $10 \mu \mathrm{m}$; tip-substrate distance: $12 \mu \mathrm{m}$; $X$ and $Y$ step sizes: $50 \mu \mathrm{m}$.

\section{Conclusions}

Alternating-current scanning electrochemical microscopy (AC-SECM) is a powerful technique for the investigation of degradation processes occurring in coated metals exposed even at very early exposures. In this case, the variation of the phase angle of the AC-current response of the electrochemical system to an AC potencial signal is employed. The measured changes in the phase angle values are related to variations in the ohmic resistance of the electrolyte volume comprised between the tip and the surface under investigation.

Chloride ions have been shown to induce a specific effect in coated samples for electrolyte uptake that result in a heterogeneous distribution of the absorbed water at the metal/coating interface, which may be regarded as the early formation of blisters under the coating.

\section{Acknowledgments}

The authors are grateful to the Ministerio de Ciencia e Innovación (Madrid, Spain) and the European Regional Development Fund (Brussels, Belgium) for supporting this work through Grant Number CTQ2009-14322. This work was initiated within the framework of the Collaborative Research Programme No. HA2006-0077 between Spain and Germany funded by the Ministerio de Educación y Ciencia (Madrid, Spain) and the German Academic Exchange Service (DAAD, Bonn).

\section{References}

1. J. E. O. Mayne, in Corrosion, vol. 2, third edn., L. L. Shreir, R. A. Jarman and G. T. Burstein, Editors, p. 14:22, Butterworths-Heinemann, Oxford (1994). 
2. J. H. W. de Wit, in Corrosion Mechanisms in Theory and Practice, P. Marcus and J. Oudar, Editors, p. 581, Marcel Dekker, New York (1995).

3. G. Grundmeier and A. Simões, in Encyclopedia of Electrochemistry, vol. 4, A. J. Bard and M. Stratmann, Editors, p. 499, Wiley-VCH, Weinheim (2003).

4. A. Forsgren, Corrosion Control through Organic Coatings, CRC Press, Boca Raton, FL (2006)

5. J. R. Macdonald, D. D. Macdonald, M. C. H. McKubrie, Editors, Impedance Spectroscopy, p. 301, Wiley, New York (1987).

6. G. Bierwagen, D. Tallman, J. Li, L. Hea and C. Jeffcoate, Prog. Org. Coat., 46, 148 (2003).

7. W. Funke, J. Oil Chem. Assoc., 62, 63 (1979).

8. H. Leidheiser Jr., Prog. Org. Coat., 7, 79 (1979).

9. W. S. Tait, Proc. of the ACS Division of Polymeric Materials: Science and Engineering, 68, 101 (1993).

10. A. J. Bard, F. F. Fan, J. Kwak and O. Lev, Anal. Chem., 61, 132 (1989).

11. A. J. Bard, F.- R. Fan and M. Mirkin, in Physical Electrochemistry: Principles, Methods, and Applications, I. Rubinstein, Editor, p. 209, Marcel Dekker, New York (1995).

12. A. J. Bard and M. V. Mirkin, Editors, Scanning Electrochemical Microscopy, Marcel Dekker, New York (2001).

13. R. M. Souto, Y. González-García, S. González and G. T. Burstein, Corros. Sci., 46, 2621 (2004).

14. R. M. Souto, Y. González-García and S. González, Corros. Sci., 50, 1637 (2008).

15. R. M. Souto, Y. González-García and S. González, Prog. Org. Coat., 65, 435 (2009).

16. R. M. Souto, Y. González-García, S. González and G.T. Burstein, Electroanal., 21, 2569 (2009).

17. R. M. Souto, Y. González-García, J. Izquierdo and S. González, Corros. Sci., 52, 748 (2010).

18. A. S. Baranski and P. M. Diakowski, J. Solid State Electrochem., 8, 683 (2004).

19. M. Etienne, A. Schulte and W. Schuhmann, Electrochem. Commun., 6, 288 (2004).

20. A. S. Baranski and P. M. Diakowski, J. Solid State Electrochem., 8, 683 (2004).

21. K. Eckhard and W. Schuhmann, Analyst, 133, 1486 (2008).

22. K. Eckhard, T. Erichsen, M. Stratmann and W. Schuhmann, Chem.-Eur. J., 14, 3968 (2008).

23. M. Pähler, J. J. Santana, W. Schuhmann and R.M. Souto, Chem.-Eur. J., 17, 905 (2011).

24. P. M. Diakowski and A. S. Baranski, Electrochim. Acta, 52, 854 (2006).

25. B. Ballesteros Katemann, A. Schulte, E. J. Calvo, M. Koudelka-Hep and W. Schuhmann, Electrochim. Acta, 123, 223 (2002).

26. B. B. Katemann, A. Schulte, E. J. Calvo, M. Koudelka-Hep and W. Schuhmann, Electrochem. Commun., 4, 134 (2002).

27. A. Schulte, S. Belger and W. Schuhmann, J. Mater. Sci. Eng. A, 378, 523 (2004).

28. C. Kranz, M. Ludwig, H. E. Gaub and W. Schuhmann, Adv. Mater., 7, 38 (1995). 